\title{
Encontrando os padrões sequenciais em apresentações orais de estudantes utilizando Sequential Pattern Mining
}

\author{
Felipe Vieira ${ }^{1}$, Cristian Cechinel ${ }^{1}$, Roberto Munoz ${ }^{2}$, Robson Lemos ${ }^{1}$, \\ Tiago Weber ${ }^{1}$ \\ ${ }^{1}$ Programa de Pós-Graduação em Tecnologias da Informação e Comunicação, \\ Universidade Federal de Santa Catarina, Araranguá, Brasil \\ ${ }^{2}$ Escuela de Ingeniería Civil Informática, \\ Universidad de Valparaíso, Valparaíso, Chile \\ felipe.roquedposgrad.ufsc.br, \{cristian,robson, Tiago\}@ufsc.br \\ roberto.munoz@uv.cl
}

\begin{abstract}
The present paper describes a sequential pattern mining based approach to identify the main corporal sequences in students oral presentations during a given course. Data from students presentations was collected through the use of Microsoft Kinect and Leikelen software, the total number of observations was 65. The 7 collected features were used as input information in the SPMF tool, allowing the identification of main sequences of presenters. Sequences with the Hands Down attribute were the most frequent in all presentations. It has also been found that the presentations 1 and 3 are more similar in terms of sequence than with the second. The evaluation of the sequences can be integrated into the tool so that the teacher can return feedback to the students about their postures.
\end{abstract}

Resumo. O presente trabalho descreve uma abordagem de Mineração de Padrão Sequencial para identificar as principais sequências corporais em apresentações orais de estudantes durante um determinado curso. Os dados das apresentações dos alunos foram coletados através do uso do Microsoft Kinect e do software Leikelen, totalizando 65 observações. As 7 características coletadas foram utilizadas como informações de entrada na ferramenta SPMF, permitindo a identificação das principais sequências dos apresentadores. Sequências com o atributo Mãos Baixas foram as mais frequentes em todas as apresentações. Verificou-se também que as apresentações 1 e 3 são mais semelhantes em termos de sequência do que com a segunda. A avaliação das sequências pode ser integrada na ferramenta para que o professor possa retornar feedback aos alunos sobre suas posturas.

\section{Introdução}

O desenvolvimento de habilidades de comunicação e trabalho em equipe tem sido indicado por organizações profissionais e recomendações de currículo e ainda por relatórios para programas de graduação universitária [Sabin et al. 2017] como expertise fundamental para a preparação de futuros profissionais, em diferentes áreas do conhecimento. Quanto às habilidades de comunicação, o objetivo principal é transmitir as informações de 
VIII Congresso Brasileiro de Informática na Educação (CBIE 2019)

Anais do XXX Simpósio Brasileiro de Informática na Educação (SBIE 2019)

forma clara e coerente para as pessoas. Bons comunicadores demonstram suas habilidades de apresentação com características verbais e não verbais, como linguagem corporal, contato visual com o público ou o espaço que ocupam no palco [York 2013]. A linguagem corporal é uma parte importante dos processos de aprendizagem e comunicação. Além disso, posturas corporais e gestos são usados em apresentações orais para transmitir ideias e mensagens [Mehrabian 2017].

Hoje, tecnologias e sensores avançados estão disponíveis em ambientes de aprendizagem que permitem a captura de dados por meio de diferentes modalidades. De acordo com Ochoa [Ochoa 2017], o campo da análise de aprendizagem multimodal (MMLA) tenta incorporar diferentes fontes de dados de aprendizagem na pesquisa de análise de aprendizagem (LA), além do tradicional arquivo de log, capturado pelos sistemas online. Tecnologias avançadas de sensores permitem capturar dados biométricos com diferentes modalidades. Isso inclui artefatos de aprendizagem, olhar, posturas, ações , expressões faciais, fala, escrita e desenho. Esses artefatos de aprendizado são exemplos de informações multimodais que foram gradualmente incorporadas a sistemas de aprendizado.

Existem alguns desafios associados à combinação de dados de várias origens, resultando em resultados ruidosos. Problemas com dados de uma variedade de fontes e programas, envolvendo reconhecimento facial e de fala, geralmente têm resoluções complexas, que geralmente são resolvidas através de técnicas de aprendizado de máquina [Ochoa et al. 2016]. Além disso, a identificação e caracterização de estudantes de graduação é um ponto de partida fundamental, a fim de se obter melhores processos de ensino e aprendizagem. Na avaliação de alunos que usam técnicas de MMLA, após a captura de dados de um grupo de usuários e diferentes fontes, os conjuntos de dados devem ser mesclados para processamento estatístico ou mineração de dados, para extrair informações razoáveis a partir dele. Assim, este trabalho procura encontrar padrões em dados sequenciais de apresentações de estudantes. A mineração de dados sequenciais é a busca dos relacionamentos entre as ocorrências de eventos sequências [Zhao and Bhowmick 2003].

O restante do artigo está organizado da seguinte forma. Na Seção 2 são apresentados os trabalhos relacionados com a identificação de padrões posturais. Já na Seção 3 é apresentada uma visão geral da metodologia e das etapas realizadas para obtenção dos resultados. Na Seção 4 são apresentados os resultados encontrados. A Seção 5 apresenta as considerações finais e propostas de trabalhos futuros.

\section{Trabalhos Relacionados}

A análise de aprendizado multimodal é um campo relacionado à LA, com ênfase na análise de modalidades de comunicação complexas durante atividades de aprendizagem, como fala, escrita e interação não-verbal [Scherer et al. 2012]. Embora relativamente jovem, o MMLA é um campo ativo e uma variedade de técnicas e aplicações tem sido propostas nos últimos anos.

Uma abordagem usual no MMLA é a integração de gravações de câmera com outros dados, para analisar o comportamento do aluno. Por exemplo, em [Bidwell and Fuchs 2011] usaram uma gravação de vídeo com múltiplas câmeras e classificações de observadores para propor uma estrutura de análise de comportamento. 
VIII Congresso Brasileiro de Informática na Educação (CBIE 2019)

Anais do XXX Simpósio Brasileiro de Informática na Educação (SBIE 2019)

Seu objetivo era modelar e classificar o engajamento do aluno a partir de sequências de alvos do olhar dos alunos, usando o software de rastreamento de rostos. Além disso, um grupo de observadores especialistas classificou os comportamentos dos alunos em categorias (engajados, atenciosos ou de transição). Com essas duas entradas, um classificador de engajamento do aluno foi criado para fornecer um relatório ao professor. Além disso, em [Ochoa et al. 2013], as informações de vídeo, áudio e traço da caneta foram usadas para discriminar entre especialistas e não especialistas em grupos de alunos que solucionam problemas matemáticos.

Outra instância de gravações em vídeo com várias câmeras que está sendo usada em contextos de sala de aula é o trabalho [Raca and Dillenbourg 2013], em que 3 a 4 câmeras foram usadas para coletar dados em uma sala de aula regular, de maneira discreta. Em seguida, informações de movimento do olhar e do corpo foram extraídas dos vídeos para fornecer um relatório ao professor.

Em [Le et al. 2013], o uso de um dispositivo Kinect foi proposto como uma alternativa para resolver problemas que de outra forma seriam difíceis de resolver usando câmeras convencionais, no reconhecimento de posturas humanas. Eles usaram a ferramenta de rastreamento do esqueleto fornecida pelo Kinect SDK para coletar informações conjuntas. Com base no esqueleto rastreado, as posições das articulações e os ângulos foram usados como características. Estes foram então utilizados na técnica da máquina de vetores de suporte (SVM) para reconhecer as posturas humanas.

Em [Echeverría et al. 2014] também usaram o dispositivo Kinect para detectar posturas comuns das articulações do corpo do esqueleto. Eles então empregaram o CMeans fuzzy para extrair generalizações de posturas, por pessoa, e então realizaram a clusterização usando o algoritmo K-Means.

Em [Reilly et al. 2018], analisaram os dados do Kinect e da fala, coletados durante uma tarefa de programação de robôs. Eles mostraram a correlação entre certos movimentos e padrões de gestos com ganhos de aprendizagem. Eles também usaram algoritmos de agrupamento para encontrar categorias recorrentes de posição do corpo, que foram então usadas para analisar a quantidade de tempo que os alunos gastaram com elas e ganhos de aprendizado.

\section{Metodologia}

\subsection{Descrição do caso}

O contexto de aprendizado do presente estudo é o curso "Introdução à Engenharia " oferecido aos estudantes de engenharia da Universidade de Valparaiso, Chile, durante o ano de 2017. Durante o curso, os alunos realizaram 3 apresentações orais em grupos sobre três tópicos previamente definidos no curso totalizando 65 observações, sendo, 20 para a primeira apresentação, 22 para a segunda apresentação e 23 para a terceira.

\subsection{Microsoft Kinect}

A captura de dados para este trabalho foi feita pelo Microsoft Kinect. É capaz de capturar até 6 pessoas simultaneamente. O kinect mostrou-se eficiente na extração dos padrões de postura e fala necessários para os experimentos. Suas vantagens estão relacionadas ao número de sensores presentes no dispositivo. Ele contém um sensor de profundidade, 
VIII Congresso Brasileiro de Informática na Educação (CBIE 2019)

Anais do XXX Simpósio Brasileiro de Informática na Educação (SBIE 2019)

uma câmera colorida e um conjunto completo de microfones que fornece recursos de reconhecimento de voz, captura facial e reconhecimento de movimentos em todo o corpo.

Usamos o rastreamento do esqueleto para classificar as posições dos alunos e a detecção de voz. Com isso, foi possível representar o corpo humano como um número de articulações. Essas articulações são partes do corpo: braços, pescoço, mãos e cabeça, por exemplo, Figura 1 mostra duas pessoas e suas representações por rastreamento do esqueleto. Essas representações de rastreamento esquelético foram utilizadas para classificar ações pré-definidas e treinadas e, assim, montar as bases de dados utilizadas. O tempo gasto em cada postura / ação é armazenado também.

\subsection{Procedimentos e coleta de dados}

Os dados capturados correspondem a apresentações orais no curso Introdução à Engenharia. O curso é oferecido no primeiro semestre aos alunos do curso de Engenharia Informática (na Universidade de Valparaiso, Chile). No curso, os alunos devem realizar três apresentações orais avaliadas, associadas a 1) desenvolvimento web, 2) microcontroladores e 3) banco de dados (com um mês entre cada apresentação). Cada apresentação foi realizada por grupos de 2 alunos, com um máximo de 5 minutos por grupo. Os slides do PowerPoint podem suportar todas as apresentações dos alunos. Todas as apresentações foram capturadas usando o software Microsoft Kinect e Leikelen. Os alunos tiveram que assinar um consentimento informado no início do curso. Além disso, este trabalho foi aprovado pelo Comitê de Ética em Pesquisa com Seres Humanos da Faculdade de Medicina da Universidade de Valparaiso.

\subsection{Ferramenta Leikelen}

Usamos o software Leikelen para coleta e processamento de dados (veja a Figura 1). O software permite detectar, armazenar e visualizar as posturas corporais das pessoas gravadas. Leikelen tem opções para adicionar posturas personalizadas, para exportar e importar cenas, e para adicionar um visualizador das posturas detectadas, juntamente com uma linha do tempo. Por fim, Leikelen também permite exportar os dados a serem visualizados usando outras ferramentas de mineração de dados.

Leikelen usa o MS Kinect para capturar posições do corpo e o algoritmo ADABoost para classificar as posturas. O software tem 10 posturas corporais pré-definidas e validadas, juntamente com outros tipos de métricas, como 1) distância proxêmica (distância entre duas ou mais pessoas), 2) se a pessoa está olhando para o público, 3) inclinação do corpo e 4) se a pessoa está falando.

\subsection{Características coletadas}

Os dados usados no presente trabalho são mostrados na Tabela 1. Os dados brutos são compostos por uma sequência de períodos e a respectiva ação / postura do aluno, capturada pelo sistema para aquele período determinado. Por exemplo, um aluno pode passar um certo período com o braço explicando, em seguida, deixar os braços para baixo por um período muito curto de tempo e, em seguida, começar a apontar para os slides, finalmente retornando à posição com o braço explicando. Para esta sequência, obtemos quatro registros compostos pelas ações / posturas capturadas (Uma Mão, Mãos Baixas, Apontando e Uma Mão), juntamente com o período que cada ação / postura durou. 
VIII Congresso Brasileiro de Informática na Educação (CBIE 2019)

Anais do XXX Simpósio Brasileiro de Informática na Educação (SBIE 2019)

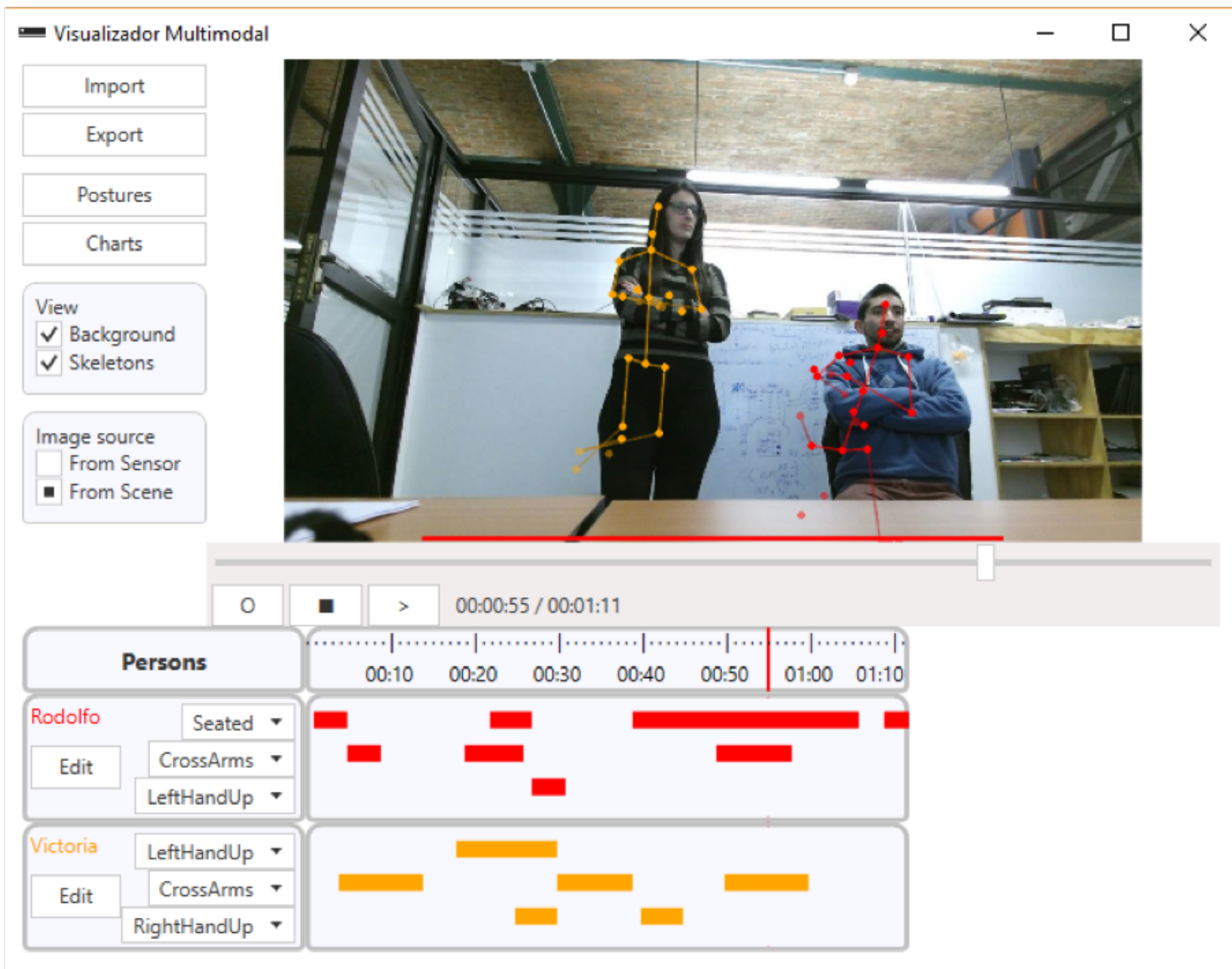

Figura 1. Tela de aplicativo Leikelen mostrando os modelos de esqueleto de duas pessoas.

Tabela 1. Descrição de cada característica da base de dados inicial

\begin{tabular}{|c|l|l|}
\hline N & Características & \multicolumn{1}{c|}{ Descrição } \\
\hline 1 & Mão na Face & O apresentador tem uma mão no queixo. \\
\hline 2 & Mão na Cabeça & O apresentador tem uma mão na nuca. \\
\hline 3 & Mãos no Quadril & O apresentador tem as mãos na cintura. \\
\hline 4 & Mãos Baixas & O apresentador tem as mãos para baixo. \\
\hline 5 & Uma Mão & $\begin{array}{l}\text { O apresentador está explicando com uma mão } \\
\text { para baixo e a outra dobrada em posição explicativa. }\end{array}$ \\
\hline 6 & Mãos Abertas & $\begin{array}{l}\text { O apresentador está explicando com as duas mãos } \\
\text { (as duas mãos com os bracos dobrados). }\end{array}$ \\
\hline 7 & Apontando & $\begin{array}{l}\text { O apresentador está apontando com uma mãos } \\
\text { (braço estendido horizontalmente). }\end{array}$ \\
\hline
\end{tabular}

\subsection{Sequential Pattern Mining}

Neste trabalho, a técnica de Sequential Pattern Mining foi utilizada. Esssa é uma ferramenta conhecida na área de mineração de dados. Em uma base de dados sequencial, cada sequência consiste em uma lista de transações [Saraf et al. 2015]. Então, o Sequential Pattern Mining trabalha para encontrar todos os padrões de sequências baseado no 
VIII Congresso Brasileiro de Informática na Educação (CBIE 2019)

Anais do XXX Simpósio Brasileiro de Informática na Educação (SBIE 2019)

suporte mínimo dado pelo usuário inicialmente.

Para a obtenção dos resultados, foi utilizado o SPMF (Sequential Pattern Mining Framework) [Fournier-Viger et al. 2016]. A ferramenta é uma biblioteca de código aberto em mineração de dados. Ela oferece mais de 120 algoritmos de mineração implementados em JAVA. A ferramenta também possui uma tela de visualização onde não é necessário trabalhar com a programação.

Inicialmente, os dados utilizados no trabalho eram sequências de strings onde essas continham os próprios atributos. Por exemplo, uma sequência poderia ser \{Mãos Baixas, Mãos Baixas, Mãos Baixas\}, para uma determinada observação. O software SPMF não aceita esse tipo de entrada para os algoritmos, devendo essa sequência ser convertida. Convertendo o atributo Mãos Baixas para o número 1 (todos os atributos foram convertidos para números), a sequência que o SPMF aceita como entrada seria $\{1$

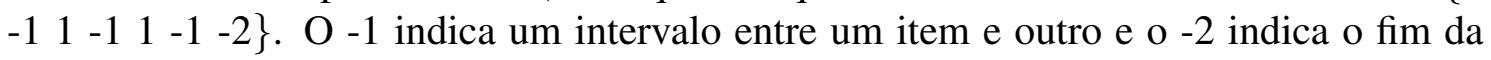
sequência da observação.

Para a obtenção dos padrões sequenciais mais frequentes foi usado o algoritmo prefixSpan, que está presente no SPMF. Os parâmetros utilizados no algoritmo foram de $60 \%$ de Minsup. O algoritmo prefixSpan, funciona da seguinte maneira:

- Encontre padrões de 1-sequência - Percorre uma base de dados sequencial $S$ uma vez encontrando todos os itens frequentes em sequências, resultando no prefixos.

- Dividir espaço de busca - O conjunto completo de padrões sequenciais pode ser particionado baseado no prefixo derivado da primeira etapa. Todos os padrões sequenciais são derivados nessa etapa.

- Encontre sub conjuntos de padrões sequenciais - Os subconjuntos de padrões sequenciais podem ser extraídos através da construção do correspondente conjunto de bases de dados projetadas, sendo cada delas construída recursivamente.

\section{Resultados - Avaliação das sequências encontradas}

Foram avaliadas as apresentações individualmente, avaliando-se o suporte (quantidade de ocorrência da sequência na apresentação) de cada uma das sequências. Neste trabalho decidiu-se analisar as 10 primeiras sequências que respeitavam suporte estabelecido. Com essas 10 primeiras sequências avaliadas é possível traçar um paralelo entre as três apresentações de modo geral.

Algumas ocorrências são individuais, sendo que não existe um atributo subsequente a este. Por exemplo, temos que a sequência (atributo) mais frequente nessa apresentação é o atributo Uma mão com um suporte de 18. Esse tipo de padrão ocorre em todas as outras três apresentações, sendo um comportamento esperado pois o algoritmo encontra ocorrências de Itens (atributos) e não necessariamente devendo conter sequências. Esse tipo de informação (ocorrências únicas) foram omitidos por não apresentarem informação que explique o comportamento sequencial desejado.

Na primeira apresentação o suporte máximo levando em conta apenas as sequencias foi de 17. Além disso, o algoritmo encontrou 202 padrões sequenciais que respeitaram o suporte mínimo estabelecido. A ferramenta levou $17 \mathrm{~ms}$ para encontrar esses padrões. O padrão que mais ocorreu foi duas ocorrências de Mãos Baixas, indicando que 
VIII Congresso Brasileiro de Informática na Educação (CBIE 2019)

Anais do XXX Simpósio Brasileiro de Informática na Educação (SBIE 2019)

o estudante passa tempo considerável com os braços baixos, esse resultado pode ser visto na Tabela 2. Este é um padrão esperado, pois ao apresentar a pessoa pode passar algum tempo com as mãos baixas.

Na sequência temos que o padrão mais frequente é os estudantes apontando duas vezes seguidas, este com suporte de 17 . Isso indica que o estudante está apontando para algo (slide, por exemplo) por algum tempo. A seguir, ainda na Tabela 2, temos que a maioria das sequências apresentam combinações dos estudantes com as mãos baixas e na posição de mãos abertas. O Item Mãos Baixas seguido de Uma Mão ocorre com suporte de 15. Isso pode estar indicando que o estudante sai de uma posição de não atividade para a atividade, explicando algo com uma das mãos. O comportamento geral das sequências continua semelhante para os demais padrões encontrados, sendo esses essencialmente combinações de posições de Mãos Baixas com Mãos Abertas.

Tabela 2. 10 maiores ocorrências de sequências na apresentação 1.

\begin{tabular}{|l|l|}
\hline \multicolumn{2}{|c|}{ Apresentação 1 } \\
\hline Padrão & Suporte \\
\hline Mãos Baixas, Mãos Baixas & 17 \\
\hline Apontando, Apontando & 17 \\
\hline Mãos Baixas, Mãos Baixas, Mãos Baixas & 16 \\
\hline Mãos Abertas, Mãos Baixas & 15 \\
\hline Mãos Baixas, Mãos Abertas & 15 \\
\hline Mãos Baixas, Mãos Abertas, Mãos Baixas & 15 \\
\hline Mãos Baixas, Mãos Baixas, Mãos Baixas, Mãos Baixas & 15 \\
\hline Mãos Baixas, Uma Mão & 15 \\
\hline Uma Mão, Mãos Baixas, Uma Mão & 15 \\
\hline Uma Mão, Mãos Abertas & 15 \\
\hline
\end{tabular}

Para a segunda apresentação o mesmo padrão que era o mais frequente na primeira apresentação também ocorre na segunda apresentação como o mais frequente, com um suporte de 20, a Tabela 3 apresenta esse resultado. Importante notar que o algoritmo encontrou apenas 24 padrões nessa apresentação em um tempo de $13 \mathrm{~ms}$. Esse numero baixo de padrões pode ser explicado pelo fato de o algoritmo não encontrar padrões sequenciais que respeitem o suporte mínimo estabelecido. Todos os 10 padrões que mais aconteceram nessa apresentação foram combinações das posições Mãos Baixas e Mãos Abertas.

Por último, na terceira apresentação, o algoritmo encontrou 222 padrões de sequências em um tempo de $35 \mathrm{~ms}$. O padrão mais frequente nesta apresentação passa a ser uma sequência das posturas de Mãos Abertas com Mãos Baixas, com suporte de 22, como mostra a Tabela 4. Diferentemente do que vinha ocorrendo, onde a sequência de Mãos Baixas era mais frequente. Isso pode indicar que nessa etapa das apresentações os estudantes já explicam mais com os duas mãos, passando a interagir mais com os braços do que no inicio.

Os padrões com os atributos Mãos Baixas e Mãos Abertas ocorrem de maneiras variadas em todas as apresentações, como pode ser visto nas tabelas 2, 3 e 4. Estes são os atributos que ocorrem com mais frequência nas sequências de todas as apresentações. 
VIII Congresso Brasileiro de Informática na Educação (CBIE 2019)

Anais do XXX Simpósio Brasileiro de Informática na Educação (SBIE 2019)

Tabela 3. 10 maiores ocorrências de sequências na apresentação 2.

\begin{tabular}{|c|c|}
\hline \multicolumn{2}{|l|}{ Apresentação 2} \\
\hline Padrão & Suporte \\
\hline Mãos Baixas, Mãos Baixas & 20 \\
\hline Mãos Abertas, Mãos Baixas & 19 \\
\hline Mãos Baixas, Mãos Baixas, Mãos Baixas & 19 \\
\hline Mãos Abertas, Mãos Abertas & 18 \\
\hline Mãos Abertas, Mãos Abertas, Mãos Abertas, Mãos Abertas & 17 \\
\hline Mãos Abertas, Mãos Abertas, Mãos Baixas & 17 \\
\hline Mãos Abertas, Mãos Baixas, Mãos Baixas & 16 \\
\hline Mãos Baixas, Mãos Abertas, Mãos Baixas, Mãos Baixas & 15 \\
\hline Mãos Abertas, Mãos Abertas, Mãos Baixas, Mãos Baixas & 15 \\
\hline Mãos Baixas, Mãos Abertas, Mãos Baixas & 15 \\
\hline
\end{tabular}

Tabela 4. 10 maiores ocorrências de sequências na apresentação 3.

\begin{tabular}{|c|c|}
\hline \multicolumn{2}{|l|}{ Apresentação 3} \\
\hline Padrão & Suporte \\
\hline Mãos Abertas, Mãos Baixas & 22 \\
\hline Mãos Abertas, Mãos Baixas, Mãos Baixas & 21 \\
\hline Mãos Baixas, Mãos Baixas & 21 \\
\hline Mãos Abertas, Mãos Abertas & 20 \\
\hline Mãos Abertas, Mãos Abertas, Mãos Baixas & 20 \\
\hline Mãos Abertas, Mãos Baixas, Mãos Baixas, Mãos Baixas & 20 \\
\hline Mãos Abertas, Mãos Baixas & 20 \\
\hline Mãos Baixas, Mãos Abertas, Mãos Baixas & 20 \\
\hline Mãos Baixas, Mãos Baixas, Mãos Abertas & 20 \\
\hline Mãos Baixas, Mãos Baixas, Mãos Baixas & 20 \\
\hline
\end{tabular}

Eles podem aparecer em sequências de varias repetições de Mãos Baixas seguidos de uma de Mãos Abertas, por exemplo.

Ainda para comparar melhor as três apresentações, são apresentadas sequências que respeitam o suporte mínimo estabelecido e ainda que ocorrem em duas ou mais apresentações. Dessa forma, mais padrões que acontecem em todas as apresentações podem ser encontrados. A Tabela 5 apresenta 6 padrões que ocorrem em pelo menos duas apresentações. Novamente fica evidente que os atributos Mãos Baixas e Mãos Abertas aparecem em todas as sequências encontradas. Esse é um comportamento facilmente explicado visto que normalmente as pessoas em uma apresentação ficam bastante tempo com os baixos estendidos para baixo, sendo que a posição Mãos Abertas pode ser vista como uma posição de mãos intermediaria.

Primeiramente, analisando a Tabela 5, percebe-se padrões esperados nas sequências dos dados. Por exemplo, temos que o apresentador estava apontando (explicando com uma das mãos) e posteriormente passa a explicar com as duas mãos (Mãos Abertas), sendo essas trocas normais em apresentações orais. Esse padrão (sequência), ocorre para as apresentações 1 e 3, não sendo observado para a apresentação 2 . 
Ocorrendo para a primeira apresentação e também para a terceira apresentação, o padrão com 3 Mãos Baixas seguidas seguido do atributo Apontando mostra o que ocorre com mais frequência na maioria das sequências avaliadas. A sequência grande de Mãos Baixas seguidos de um outro atributo. Este pode ser considerado um comportamento normal, onde o estudante deve passar um maior tempo com as mãos baixas, visto que são apresentações em duplas. Um padrão similar pode ser visto também em uma sequência que ocorre para todas as apresentações, 3 vezes de Mãos Baixas seguidos de um Mãos Abertas.

Uma importante diferença entre a segunda apresentação e as outras duas apresentações (primeira e terceira) é que na segunda apresentação o algoritmo encontrou sequências com apenas três atributos, Mãos Baixas, Mãos Abertas e Uma mão. Na primeira e na terceira apresentação outros atributos também apareceram nas sequências, como a sequência Mãos Baixas,Mãos na Face que ocorre com suporte 12 para a primeira apresentação.

Tabela 5. Sequências iguais entre apresentações.

\begin{tabular}{|l|l|}
\hline \multicolumn{1}{|c|}{ Padrão } & Apresentações \\
\hline Apontando, Mãos abertas & 1,3 \\
\hline Apontando, Mãos Baixas & 1,3 \\
\hline Apontando, Mãos Baixas, Mãos Abertas & 1,3 \\
\hline Mãos Baixas, Mãos Baixas, Mãos Baixas, Mãos Baixas, Apontando & 1,3 \\
\hline Mãos Baixas, Apontando, Mãos Baixas & 1,3 \\
\hline Mãos Baixas, Mãos Baixas, Mãos Baixas, Mãos Abertas & $1,2,3$ \\
\hline
\end{tabular}

\section{Considerações finais}

O MMLA pode ajudar na avaliação de ambientes complexos de aprendizado usando dados de várias fontes. No presente estudo, verificamos que, como esperado, o atributo mais presente nas sequências foi o Mãos Baixas. Também ficou evidente que a primeira e a terceira apresentações são mais parecidas em termos de sequência do que com a segunda apresentação. Existem ainda muitas sequências envolvendo os atributos Mãos Baixas e o atributo Mãos Abertas, onde eles podem aparecer variadas vezes em sequência.

Para trabalhos futuros, novas comparações devem ser realizadas em diferentes grupos de estudantes. Esses novos estudantes viriam de diferentes áreas de conhecimento, como engenharia e saúde. Essa distinção pode mostrar comportamentos diferentes entre as áreas do conhecimento. Ou pode revelar comportamentos semelhantes entre eles. Aliado a isso, técnicas de visualização de informação podem ser exploradas para auxiliar na detecção de padrões obtidos pelos alunos, nas diferentes áreas do conhecimento. Como forma de retornar o feedback dos alunos, os padrões de sequência podem ser integrados ao software para visualização das apresentações em tempo real. Espera-se também testar outras técnicas de Sequential Pattern Mining para melhorar a avaliação do problema.

\section{Referências}

Bidwell, J. and Fuchs, H. (2011). Classroom analytics: Measuring student engagement with automated gaze tracking. Technical report, University of North Carolina at Chapel Hill, Department of Computer, Chapel Hill, NC. 
VIII Congresso Brasileiro de Informática na Educação (CBIE 2019)

Anais do XXX Simpósio Brasileiro de Informática na Educação (SBIE 2019)

Echeverría, V., Avendaño, A., Chiluiza, K., Vásquez, A., and Ochoa, X. (2014). Presentation skills estimation based on video and kinect data analysis. In Proceedings of the 2014 ACM Workshop on Multimodal Learning Analytics Workshop and Grand Challenge, MLA '14, pages 53-60, New York, NY, USA. ACM.

Fournier-Viger, P., Lin, J. C.-W., Gomariz, A., Gueniche, T., Soltani, A., Deng, Z., and Lam, H. T. (2016). The spmf open-source data mining library version 2. In Joint European conference on machine learning and knowledge discovery in databases, pages 36-40. Springer.

Le, T., Nguyen, M., and Nguyen, T. (2013). Human posture recognition using human skeleton provided by kinect. In 2013 International Conference on Computing, Management and Telecommunications (ComManTel), pages 340-345.

Mehrabian, A. (2017). Nonverbal Communication. Routledge.

Ochoa, X. (2017). Multimodal Learning Analytics. In Lang, C., Siemens, G., Wise, A. F., and Gaševic, D., editors, The Handbook of Learning Analytics, pages 129-141. Society for Learning Analytics Research (SoLAR), Alberta, Canada, 1 edition.

Ochoa, X., Chiluiza, K., Méndez, G., Luzardo, G., Guamán, B., and Castells, J. (2013). Expertise estimation based on simple multimodal features. In Proceedings of the 15th ACM on International Conference on Multimodal Interaction, ICMI '13, pages 583590, New York, NY, USA. ACM.

Ochoa, X., Worsley, M., Weibel, N., and Oviatt, S. (2016). Multimodal learning analytics data challenges. In Proceedings of the Sixth International Conference on Learning Analytics \& Knowledge - LAK16. ACM Press.

Raca, M. and Dillenbourg, P. (2013). System for assessing classroom attention. In Proceedings of the Third International Conference on Learning Analytics and Knowledge, LAK' 13, pages 265-269, New York, NY, USA. ACM.

Reilly, J., Ravenell, M., and Schneider, B. (2018). Exploring collaboration using motion sensors and multi-modal learning analytics. In Proceedings of the 11th International Conference on Educational Data Mining.

Sabin, M., Alrumaih, H., Impagliazzo, J., Lunt, B., Zhang, M., Byers, B., Newhouse, W., Paterson, B., Peltsverger, S., Tang, C., et al. (2017). Curriculum guidelines for baccalaureate degree programs in information technology. Technical report, Technical Report. ACM, New York, NY, USA.

Saraf, P., Sedamkar, R., and Rathi, S. (2015). Prefixspan algorithm for finding sequential pattern with various constraints. International Journal of Applied Information Systems (IJAIS), pages 2249-0868.

Scherer, S., Worsley, M., and Morency, L. (2012). 1st international workshop on multimodal learning analytics. In ICMI'12 - Proceedings of the ACM International Conference on Multimodal Interaction.

York, D. (2013). Investigating a Relationship between Nonverbal Communication and Student Learning. PhD thesis, Lindenwood University.

Zhao, Q. and Bhowmick, S. S. (2003). Sequential pattern mining: A survey. ITechnical Report CAIS Nayang Technological University Singapore, 1:26. 